\title{
Mediale Hybridisierung in Hernando Alvarado Tezozomocs „Crónica Mexicana“
}

\author{
Annegret Richter (Leipzig)
}

\begin{abstract}
The Crónica Mexicana is an account of the Aztec history by Mexica historian Hernando Alvarado Tezozomoc. Written ca. 1598, the chronicle focuses on the journey of the Aztec people, on its mythical origins in Aztlan, its migrations and its military endeavors until becoming the most important power in central Mexico. The work ends with the arrival of the Spaniards in Tlaxcala. In this paper, I analyse how Tezozomoc narrates Aztec history by combining narrative strategies of both Spanish and Aztec origins. I argue that the chronicle can not only be read as an example of the hybridization of different cultural traditions but that it also presents a new understanding of the Spanish colonization by integrating it into a Mesoamerican concept of time.
\end{abstract}

\section{$1 \quad$ Einleitung}

Die Crónica Mexicana wurde um 1598 von Hernando Alvarado Tezozomoc verfasst und behandelt die Geschichte der Mexica, eine indigene Gruppe, die landläufig unter dem Ethnonym Azteken erfasst wird, von deren mythischen Urzeiten bis zur Ankunft der Spanier unter Hernán Cortés in Tlaxcala. Von Interesse ist der Text nicht nur für die Erschließung der aztekischen Geschichte, sondern auch für die Verhandlung von Überlieferungstraditionen im Kontext des spanischen Kolonialismus.

Ich möchte im Folgenden zeigen, dass die Crónica Mexicana von den aztekischen Überlieferungsformen der Piktographien und der oralen Tradierung geschichtlichen Wissens geprägt ist und erklären, welche Konsequenzen das für die Vertextungsstrategien dieser in spanischer Sprache verfassten Chronik hat. Nach einer knappen Vorstellung von Autor und Werk möchte ich in einem zweiten Schritt auf die Merkmale der aztekischen Geschichtsüberlieferung eingehen, um auf dieser Grundlage herauszuarbeiten, wie diese Formen der Geschichtsschreibung in die Crónica Mexicana übersetzt werden.

\section{$2 \quad$ Autor und Werk}

Über Don Hernando de Alvarado Tezozomocs Biographie ist nicht viel bekannt. Wahrscheinlich wurde er in den 1520er Jahren in México-Tenochtitlan geboren und verbrachte dort auch sein Leben (cf. Romero Galván 2003: 6, 87). Er stammt aus einer adligen Familie des Mexi- 
ca-Adels. Seine Mutter war eine Tochter, sein Vater ein Neffe des tlatoani (,Herrscher") Moctezuma Xocoyotzin (Tezozomoc 2004: 315, 39-40). ${ }^{1}$

Über Tezozomocs Bildungsweg und Leben ist nicht viel überliefert. In der Forschung ist umstritten, ob er beispielsweise Schüler am renommierten Colegio de Santa Cruz de Tlatelolco war, das unter franziskanischer Führung für die Ausbildung der männlichen Kinder und Jugendlichen des indigenen Adels eingerichtet wurde.

Auch das genaue Datum des Todes Tezozomocs ist nicht bekannt. Aus der Crónica Mexicayotl, einem seiner Werke, ${ }^{2}$ kann man jedoch ableiten, dass er nach 1609 gestorben sein muss (cf. Tezozomoc 2004: 39; Díaz Migoyo 2001: 24). Der Herausgeber der Crónica Mexicana, Díaz Migoyo (1993: 287), erklärt die Unklarheiten über Tezozomocs Leben damit, dass dieser sich vor allem in einem indigen geprägten Umfeld bewegte, wenig Kontakt zu Spaniern hatte und daher kaum aktenkundig wurde.

Die Crónica Mexicana behandelt den Transformationsprozess der Mexica vom armen, nomadischen zum sesshaften Volk, das die Vorherrschaft über Zentralmexiko innehat. Dieser Prozess wird auf das Wirken des Gottes Huitzilopochtli zurückgeführt, der die Mexica auf ihrer Wanderung bis hin zur späteren Hauptstadt Tenochtitlan anführte und zur Kriegsführung, zur Gründung von Siedlungen und zur Ausdehnung der Herrschaft ermutigte (cf. Tezozomoc 2001: 56-57, 59, 60-64), aber auch ein eifersüchtiger Gott ist, der die Unterordnung der Mexica erwartet (cf. ibd.: 60).

Tezozomoc beschreibt die Siedlungsaktivitäten sowie die Regierungszeiten und Eroberungen von Herrschern wie Itzcoatl, Moctezuma, Ahuitzotl und anderen. Die militärischen Auseinandersetzungen mit anderen Gruppen nehmen einen bedeutenden Teil des Textes ein, etwa der Krieg mit den Tepaneken und damit der Kampf um die Vorherrschaft in Zentralmexiko. Auch dieser wird von Huitzilopochtli angeregt: „Hea, padres, hermanos mexicanos, esforçaos y hazed lo que os mandan estos tepanecas y su rrey Teçoçomoctli, que el secreto de este misterio yo lo sé. [...] con estos mandos los compramos como a esclauos, <que> lo serán en tiempo adelante“ (Tezozomoc 2001: 63-64).

Die Geschichte der Mexica beginnt an den mythischen Herkunftsorten Aztlan bzw. Chicomoztoc. Erst im Verlauf der Migrationen wechselt die Perspektive von der mythischen zur politischen Geschichte. Wie Romero Galván (2007: 173-174) feststellt, handelt es sich um eine Geschichtsschreibung, die auf der Idee einer glorreichen Entwicklung (devenir glorioso) der Mexica auf der Grundlage einer endlosen Kette von Kriegen und Eroberungen basiert. Neben der militärischen und politischen Geschichte beschreibt die Crónica Mexicana aber auch detailliert die Alltagskultur der Mexica in unterschiedlichsten Bereichen, so dass der Text auch aus ethnographischer Perspektive von Interesse ist (cf. Díaz Migoyo 1993: 287; Carrasco 1996: 20).

\footnotetext{
${ }^{1}$ Zu den Vorfahren Tezozomocs cf. Romero Galván (2003: 82); Dyckerhoff (1970: 1); Tezozomoc (2004: 276277, 330-331).

2 Die genauen Anteile der Autorschaft Tezozomocs an der Crónica Mexicayotl sind in der Forschung umstritten; zu dieser Diskussion cf. Dyckerhoff (1970: 6-8); Díaz Migoyo (1993: 288); Riese (2004: 16); Romero Galván (2007: 173); Schroeder (2011).
} 
Tezozomocs Chronik endet mit der Ankunft der Spanier unter Hernán Cortés in Tlaxcala, einer mit den Mexica verfeindeten Region (die Eroberung durch die Spanier wird also nicht mehr erzählt) sowie mit der Ankündigung, die Geschichte in einem anderen Heft fortzusetzen: „Y a esto, cada día tenía Monteçuma abiso de lo que pasaua <en> los caminos y como quedauan en Tlaxcala, y hizo llamamientos de todos los prençipales de sus comarcas para hazer acuerdo y cabildo, como adelante se dirá en otro cuaderno“ (Tezozomoc 2001: 484). Allerdings ist diese Fortsetzung nicht überliefert.

\section{Charakteristika der aztekischen Geschichtsüberlieferung}

Die Azteken tradierten ihr kulturelles Wissen in mündlicher und in piktographischer Form (Gruzinski 1993: 9). Mündliche Überlieferungen konnten in zwei grundlegende Formen gegliedert werden (ibd.): cuicatl (Gesänge, Hymnen, Gedichte) und tlahtolli (Reden, Berichte, Erzählungen). Tlahtolli war ein Oberbegriff für unterschiedliche inhaltliche Unterformen wie etwa teohtlahtolli (Religion, Kosmogonie) oder huehuetlahtolli (,alte Worte‘ zu unterschiedlichen Themen). Beide Formen folgten stilistischen Konventionen und verwendeten unterschiedliche Stilmittel, wie etwa im Falle des tlahtolli Diphrasen, Parallelismen und Prädikatsanhäufungen um ein Subjekt (cf. ibd.: 10).

Der Großteil der präspanischen Schriftquellen Mesoamerikas ist nicht erhalten. ${ }^{3}$ Bei erhaltenen frühen piktographischen Codices, wie etwa dem Codex Boturini (Tira de la peregrinación), dem Codex Borbonicus (Ritualkalender) sowie der Matrícula de Tributos, ist die genaue Datierung umstritten und es kann nicht eindeutig bestimmt werden, ob sie vor oder nach der Eroberung Mexikos (ab 1521) entstanden sind.

Die überlieferten historischen Texte Zentralmexikos lassen sich zum Teil bestimmten indigenen Gruppen zuordnen, hauptsächlich den Chalca, den Tenochca (< Tenochtitlan), den Tlaxcalteken, den Acolhuaque (< Texcoco) und den Tlatelolca (Pastrana Flores 2004: 10). Die Crónica Mexicana gehört der Tenochca-Tradition an. In der Forschung wird seit Barlow (1945) von einer auf Nahuatl geschriebenen Crónica $X$ ausgegangen, die diese Traditionslinie begründet habe, aber nicht überliefert ist. Die Annahme der Crónica $X$ soll die inhaltlichen Gemeinsamkeiten so unterschiedlicher Geschichtswerke wie der Crónica Mexicana von Tezozomoc (ca. 1598), der Historia de las Indias de la Nueva España e Islas de la Tierra Firme von Fray Diego Durán (ca. 1581) oder des Códice Aubin (ca. 1576) erklären (cf. Pastrana Flores 2004: 11-12). Im Unterschied zu den beiden spanischen Chroniken von Tezozomoc und Durán besteht der Códice Aubin aus Bildern und aus Texten auf Nahuatl und verweist damit auf die piktographische Tradition der aztekischen Geschichtsüberlieferung.

Um zu zeigen, wie aztekische Überlieferungsformen die Schreibweise der Crónica Mexicana beeinflusst haben, sollen zunächst deren zentrale Merkmale genannt werden. Dabei ist festzuhalten, dass die Rezeption der piktographischen Codices von mündlichen Überlieferungen begleitet wurde. Piktographien alleine vermitteln also nur ein ausschnitthaftes Bild ihres Gegenstandsbereichs.

\footnotetext{
${ }^{3}$ Insbesondere archäologische Quellen sind daher von großer Bedeutung für die Erforschung der vorkolonialen Geschichte Mesoamerikas. Dass sie im vorliegenden Artikel nicht besprochen werden, soll nicht ihre Relevanz schmälern, sondern ist ausschließlich der thematischen Schwerpunktsetzung des vorliegenden Artikels geschuldet.
} 
Das Schreiben (und Lesen) der Piktographien war einer kleinen Elite von Gelehrten (tlamatinime, Sg. tlamatini < mati, wissen') vorbehalten. Die Schreiber wurden tlacuiloque (Sg. tlacuilo) genannt.

Das Schreibsystem der Azteken bestand aus Piktogrammen (Repräsentationen von Personen, Objekten oder Handlungen), Ideogrammen (Konzepte, die mit Objekten verbunden werden: bspw. kann ein Pfeil für Krieg stehen, Fußspuren bezeichnen Bewegung im Raum), phonetischen Zeichen (zum Beispiel Lokativsuffixe wie -pan, -tlan, -tzin, oft in Verbindung mit toponymischen Glyphen), Zeichen für semantische Determinativa (Interpretationshilfen) und Hilfszeichen wie etwa graphische Verbindungen unterschiedlicher Elemente (cf. Gruzinski 1993: 11; Pardo López et al. 2002: 84-86).

Bildliche Elemente wurden als Oberfläche dargestellt; sollte Perspektive vermittelt werden, so wurden nahe Elemente im unteren Bildbereich angeordnet, entferntere Elemente im oberen. Freie Stellen wurden mit symbolischen Zeichen gefüllt. ${ }^{4}$ Jedes Bild wurde mit einem Rahmen versehen und stellte eine Szene dar, deren Aneinanderreihung als Erzählung gelesen werden konnte (cf. Pardo López et al. 2002: 87).

Die Spanier des 16. Jahrhunderts stuften die Piktographien unterschiedlich ein. Während einige die Bedeutung von Farben und Linien sowie der Anordnung von Zeichen etc. nicht verstanden und die Dokumente irreführend als pinturas bezeichneten (cf. Gruzinski 1993: 12), war anderen durchaus bewusst, dass es sich bei den Piktographien um die Bücher der Nahuas handelt, die nur anderen Schreibkonventionen folgten als die europäischen Bücher (Hill Boone 2000: 28).

Nach Lockhart (1992: 326) kann davon ausgegangen werden, dass „,no strict demarcation existed between writing and painting". So kann das Verb icuiloa (> tlacuilo, s. o.) mit ,malen“ wie auch mit ,schreiben` übersetzt werden (ibd.). Auch Gruzinski betont, dass

[t]he ,paintings' are images as much as texts, and words cannot offer the exact equivalent of an image. In other words, it appears that writing down pictographic knowledge necessarily involved a loss of substance, a loss all the more disquieting as it was irremediable and ineffable.

(Gruzinski 1993: 52-53)

Das Verhältnis zwischen Piktographien und mündlicher Überlieferung ist ähnlich komplex: Einerseits unterstützten die Piktographien den mündlichen Diskurs, aber zugleich war dessen Kenntnis nötig, um die Bilder (die zugleich auch Text waren) zum „Sprechen“ zu bringen. Sie waren also weit mehr als nur Gedächtnisstützen (cf. Gruzinski 1993: 13).

Im Zuge der spanischen Kolonisierung wurden viele Codices vernichtet. Bereits 1521 wurde das Archiv von Texcoco, einer der Hauptstädte des aztekischen Dreibundes, zerstört. Ab 1525, nachdem die Franziskaner den Einheimischen die öffentliche Religionsausübung untersagt hatten, zerstörten die Spanier im Tal von Mexiko und in Tlaxcala systematisch alle Tempel und vernichteten alle Piktographien, die verdächtig erschienen, dem Katholizismus zu widersprechen (cf. Gruzinski 1993: 14).

\footnotetext{
${ }^{4}$ In kartographischen Geschichtswerken kann leerer Raum auch anzeigen, dass in einem bestimmten Zeitraum nichts Erwähnenswertes passiert ist - ,empty moments in a story“, wie es Hill Boone (2000: 63) nennt.
} 
Unter den Bedingungen der Klandestinität wurden noch etwa 50 Jahre lang Piktographien hergestellt. Auch die mündliche Überlieferung wurde nach der Eroberung weiter gepflegt, so dass sich das dritte mexikanische Konzil noch 1585 genötigt sah, die Gesänge der alten Religion und Kultur zu verbieten (cf. Gruzinski 1993: 18).

Aus den erhaltenen Piktographien geht Gruzinski (1993: 17) zufolge hervor, dass präspanische Zeichen- und Schreibtechniken noch in hohem Maße vermittelt wurden, so dass es nicht immer einfach ist, die Dokumente der vorkolonialen oder der kolonialen Zeit zuzuordnen. Auch kann nicht immer davon ausgegangen werden, dass nach der Eroberung entstandene Codices ausschließlich die Gegebenheiten der vorkolonialen Zeit reflektieren: „We generally forget that the vast majority of the accounts of the prehispanic world that we have were produced in the exceptional post-conquest circumstances and reflect them as much as the societies that had disappeared“" (ibd.: 32).

Zudem änderte sich mit der Zeit die Funktion der nach der Eroberung entstandenen Piktographien: So dienen sie oft der Darstellung der Besitztümer in historischen Zeiten, um gegenwärtige Ansprüche auch von zum Christentum konvertierten Personen gegenüber den Spaniern zu legitimieren. Dabei waren zwar die Unterschiede zwischen verbotenen religiösen Bilderhandschriften und legitimen historischen oft gering (cf. Gruzinski 1993: 19), in Gerichtsprozessen wurden diese Dokumente aber auch als Beweismaterial anerkannt (Hill Boone 2000: 28).

Nicht nur die Funktion, auch die Zeichentechniken änderten sich unter kolonialem Einfluss. Der Lienzo de Tlaxcala (ca. 1550-64) ist beispielsweise ein Codex, der indigene piktographische mit europäischen zeichnerischen Elementen verknüpft: Trotz des europäischen Einflusses und des kolonialzeitlich bedingten Inhalts gehört sie hinsichtlich der Piktogramme, der Perspektive und der Stilisierung zur indigenen Tradition (eine genauere Erklärung zu dieser Form der kulturellen Hybridisierung findet sich bei Gruzinski [1993: 21-23]).

Die Hybridisierung der unterschiedlichen kulturellen Tradierungsformen ist aber nicht nur in mesoamerikanischen, sondern auch in europäischen Dokumenten zu finden (etwa im Werk Sahagúns). Mit Tezozomocs Crónica Mexicana soll im Folgenden ein alphabetschriftliches Dokument näher dargestellt werden, das als Ergebnis kultureller Übersetzungsprozesse ausgehend von aztekischen Überlieferungsformen angesehen werden kann.

\section{Mediale Hybridisierung bei Tezozomoc}

Hernando Alvarado Tezozomoc ist als Angehöriger des Mexica-Adels mit den präspanischen Tradierungsformen inhaltlich wie formal ebenso vertraut wie mit der europäischen Form der Chronik. Diese doppelte kulturelle Kompetenz ermöglicht es ihm, sowohl mündliche Überlieferungen als auch piktographisches Schreiben in seinen Text zu integrieren. Die Repräsentation der Piktographien zeigt sich an zwei Verfahren: deren Thematisierung als Quellen durch die historischen Akteure, die Tezozomoc auftreten und sprechen lässt, sowie - und hier wäre der kulturelle Übersetzungsprozess im engeren Sinne zu verorten - durch den Einfluss der Piktographien auf die Textstruktur der Crónica Mexicana. 


\subsection{Die Fiktionalisierung mündlicher Überlieferungsformen}

Tezozomoc integriert Dialoge und Ansprachen in seinen Text, die ich als Konstruktion mündlich überlieferten Wissens der Mexica betrachten möchte. Der Chronist bemüht sich nämlich, die sprachlichen Konventionen der Nahua-Rhetorik beizubehalten (cf. Lockhart 1992: 390), etwa durch Formen der Anrede oder des höflichen Sprechens. So wird der Thronfolger Huitzilihuitl von den Mexica als Sohn und geliebter Enkel angesprochen, während sich die Untertanen als Eltern und Großeltern bezeichnen: „Hijo y n<uest>ro muy querido nieto“ (Tezozomoc 2001: 65), ,Hijo y nieto $\mathrm{n}<$ uest>ro tan querido de nosotros $\mathrm{u}<$ est>ros padres y abuelos“ (ibd.: 68). Auch der Dialog zwischen Tezozomoc, Herrscher von Azcapotzalco, und den Mexica folgt diesen Konventionen:

Hijo, nieto n<uest>ro muy querido, obedesçido de nosotros los miserables mexicanos, y nosotros, $\mathrm{u}<$ uest $>$ ros padres y abuelos somos, $\mathrm{y}$ en tal os tenemos y ternemos siempre, aguardando sienpre $\mathrm{u}<$ uest $>$ ros rreales mandam $<$ iento $>\mathrm{s}<\mathrm{en}>$ lo que nos fuere mandado, benimos con mucha umildad y os suplicamos por el alto balor y señorío u<uest $>$ ro, miserables de nosotros, y de $\mathrm{u}<$ uest $>$ ro basallo que está y guarda y rrige $\mathrm{u}<$ uest $>$ ra rrepública y pueblo mexicano, teniendo como tenéis esmeraldas y piedras preçiosas y tan queridas hijas $\mathbf{u}<$ uest $>$ ras. Pobre de $u<$ uest $>$ ro basallo, pues no tenemos a dónde yr ni acudir sino es a bos como a $n<u e s t>$ ro amo y señor y nosotros $\mathrm{u}<$ uest $>$ ros basallos, nos hagáis tanta merçed e mandarnos dar una hija y esmeralda y querida buestra para que baya a rregir y gouernar $u<$ uest $>$ ro pueblo mexicano y ser conjunta persona de Huitzilihuitl, $\mathrm{u}<\mathrm{uest}>$ ro leal sieruo y basallo, $\mathrm{n}<$ uest $>$ ro rrey y señor.

(Tezozomoc 2001: 66)

Bei dieser systematischen Inversion von Verwandtschaftsbegriffen handelt es sich um übliche Höflichkeitsformeln der Nahuas (cf. Lockhart 1991: 75). Die Ansprachen in der Crónica Mexicana

prove to follow Nahuatl conventions through the barrier of language and indeed at the expense of idiomatic Spanish. Tezozomoc wrote fluent if not elegant Spanish, but the speeches often have a strange ring because of the literal translation of Nahuatl idioms. Thus the polite inversions are retained; superiors call aids their fathers, inferiors call the king their grandchild, and whole addresses follow Nahuatl conventional forms.

(Lockhart 1992: 390)

Allerdings fällt auch auf, dass Tezozomoc die spanischen Bezeichnungen für politische Einheiten oder Ämter benutzt. So spricht er im obigen Zitat von „,rrepública“, „,basallos“ sowie vom ,,rrey y señor“. Der Nahuatl-Terminus für den Herrscher wäre allerdings tla(h)toani gewesen, (,der, der spricht/regiert', eine Ableitung von tla ,etwas' + (h)itoa ,sprechen'). Tezozomoc übersetzt also die politischen Termini ins Spanische, behält aber die idiomatischen Konventionen des Nahuatl bei, um so die politische Rhetorik der Mexica mimetisch repräsentieren zu können (cf. Graham Classen 1998: 71) und zugleich für einen des Nahuatl nicht mächtigen Leser verständlich zu bleiben. Allerdings wird es diesem Leser erschwert, den spezifischen Charakter des Herrschaftsamtes zu erkennen, da Tezozomoc den Vergleich mit der europäischen Monarchie durch den Terminus rrey nahelegt.

Hier wird eine Schwäche des mimentischen Verfahrens Tezozomocs deutlich: Da es nicht möglich ist, die politische Kommunikation gleichzeitig vollständig ins Spanische zu übersetzen und alle kulturellen Spezifika des politischen Systems der Nahuas auf Spanisch zu repräsentieren, kommt es in der Übersetzung zu einem Bedeutungsverlust, da nicht mehr deutlich wird, dass das Amt des tlatoani nicht in derselben Weise funktioniert wie das Amt eines eu-

ISSN 1615-3014 
ropäischen Monarchen. Dieser Umstand könnte durch metanarrative Kommentare ausgeglichen werden, auf die Tezozomoc jedoch verzichtet.

Allerdings nimmt Tezozomoc die Übersetzung von Titeln oder Ämterbezeichnungen nicht konsequent vor. Zwar werden auch die Adligen nicht als pipiltin (Sg. pilli) bezeichnet, sondern als prençipales. Jedoch behält Tezozomoc den Terminus teuctli (Pl. teteuctin) als Bezeichnung für einen Angehörigen der Oberschicht, der militärische Verantwortung trug, bei, meist als Namensbestandteil oder -ergänzung: „Hijo n<uest>ro, amado y caro nieto Ahuitl teuctli, rrey de los mexicanos, despidamos a estos prençipales de Huexoçingo, Cholula, yopitzincas, <que> se bayan a la buena bentura [...]“ (Tezozomoc 2001: 283). Für den Begriff teuctli liefert Tezozomoc in zwei Fällen en passant eine Übersetzung: „Tornó a boluerse Huiznahuatl teuctli, capitán, dixo a los balerosos mexicanos“ (ibd.: 232; sowie in ähnlicher Form ibd.: 132).

Eine zweite Form des Einbezugs mündlichen Wissens ist, neben fiktionalisierter Mündlichkeit in Form von Dialogen oder Ansprachen, der Einbezug mündlicher Quellen. Tezozomoc beruft sich immer wieder auf Äußerungen anderer Personen. Diese Verweise werden jedoch in der Regel von Tezozomoc nicht konkretisiert, wie an Formeln wie ,dizen (que)“ oder „asegún dizen“ (Tezozomoc 2001: 61; 199) deutlich wird.

Der Einbezug mündlicher Überlieferung kann aber auch aus struktureller Perspektive betrachtet werden. Graham Classen (1998: 67) zufolge steht der Einfluss oraler Überlieferung in der Crónica Mexicana in direktem Widerspruch zu den „estructuras narrativas cognoscitivas españolas“, denn Tezozomoc „basa la estructura narrativa de su crónica en los aspectos visuales y dialogados de esta tradición histórica náhuatl, y trata de captar, por medio de la lengua española, la imagen y las modalidades de la tradición oral“.

Romero Galván (2007: 175-176) differenziert drei Strategien, wie sich Oralität in Tezozomocs Text niederschlägt: durch direkte Zeugnisse (Zitate), die als oral überliefert ausgegeben werden, durch Formelsprache, die auf Mnemotechniken verweist, wie sie für orale Überlieferung typisch sind, sowie durch einen „,discurso cuyo carácter es evidentemente oral y que sólo pudo haber sido conservado por la memoria“.

Mit der ersten Strategie bezieht sich Romero Galván auf den bereits erwähnten Verweis auf mündliche Quellen. Da diese jedoch von Tezozomoc nicht kontextualisiert oder näher erläutert werden, kann nicht automatisch auf die Mündlichkeit der Überlieferung dieser Passagen geschlossen werden, wie Romero Galván (2007: 176) annimmt.

Die von Romero Galván angeführte formelhafte Sprache, die auf Doppel- bzw. Mehrfachausdrücken basiert, findet sich im Text immer wieder. Dabei setzt Tezozomoc oft Synonymformeln ein: „La benida que hizieron y tiempos y años que estubieron en llegar a este Nueuo $\mathrm{Mu}<\mathrm{n}>$ do, adelante se dirá“ (Tezozomoc 2001: 53), ,Y al tiempo que llegaron a esta çiudad abían andado y caminado muchas tierras“ (ibd.: 54), „No es a mi cargo ni boluntad que tales ofiçios y cargos tenía mi hermana Malinalxoch“ (ibd.: 56), „En muchos pueblos y gentes [...]“ (ibd.: 56). Mitunter handelt es sich aber auch um reiterative Formeln, die nicht völlig synonym, aber bedeutungsähnlich sind (cf. Romero Galván 2007: 180): „determinó y les habló su dios“ (Tezozomoc 2001: 53), ,Huitzilopochtli, ydolo dios de ellos“ (CMX 53, 1r), ,,mi prençipal benida es guerra y armas, arco y flechas“ (Tezozomoc 2001: 56), „,Todo lo tengo de 
beer y tener, pues es mi mandado y mi ofiçio“ (ibd.: 57). Darüber hinaus setzt Tezozomoc Formeln ein, die von der aztekischen Diphrase abgeleitet zu sein scheinen, wie etwa im folgenden Beispiel: „tierra y casa antigua llaman oy día Chicomoztoc, que dize Casa de siete cueuas cabernosas“ (ibd.: 53). Die sprachlichen Doppelformen, die in mündlicher Überlieferung eine mnemotechnische Funktion erfüllten, verleihen dem Text einen reiterativen Charakter (cf. Romero Galván 2007: 180). Máynez (2002: 238) definiert das Stilmittel der Diphrase folgendermaßen: „los difrasismos [...] son una figura distintiva del náhuatl - aunque no privativa de esa lengua - y se constituyen mediante la yuxtaposición de dos lexemas que se unen para formar un significado diferente del que detenta cada uno de ellos por separado“. León-Portilla (1983: 53) zufolge kamen Diphrasen nicht nur in der Dichtung, sondern weit häufiger noch im Bereich der huehuetlatolli zum Einsatz. Damit können sie als ein Stilmittel der oralen historischen Überlieferung gelten, das von Tezozomoc ins Medium der spanischen Chronik übersetzt wird.

Bei den verschiedenen Formen von Häufungsformeln der aztekischen Tradition handelt es sich um feststehende Wendungen mit mnemotechnischer Funktion, die nur noch in die Reden eingefügt werden müssen (cf. Romero Galván 2007: 181). Dieses Verfahren wird bei Tezozomoc bspw. in einer Rede von Huitzilopochtli bei den Wanderungen der Mexica deutlich. Die Rede kann Romero Galván zufolge als Verbalisierung dessen angesehen werden, was in den Piktographien nur durch eine Art Sprechblase angezeigt wird und im Rahmen der ursprünglichen Tradierung mündliche Kommentare erforderte, um verständlich zu sein (ibd.: 179-180). Huitzilopochtli bedient sich in seiner Ansprache zahlreicher Doppel- bzw. Häufungsformeln:

No es a mi cargo ni boluntad que tales ofiçios y cargos tenía mi hermana Malinalxoch desde la
salida hasta aquí, e cómo asimismo también fue yo manda $<\mathrm{d}>$ o de esta benida, que mi prençipal
benida es guerra y armas, arco y flechas, rrodelas se me dio por cargo traer, y mi oficio es gue-
rra, y yo asimismo con mi pecho, cabeça, braços <en $>$ todas partes tengo de uer y ser mi oficio.
En muchos pueblos y gentes que oy ay tengo de estar por delante y fronteras y aguardar gentes
de diuersas naçiones, y e de sustentar y dar de comer y beuer, y allí les tengo de aguardar y jun-
tallos de todas suertes de naçiones; y esto no graçiosamente. Primero e de conquistar en guerras
para tener y nombrar mi casa de preçiada esmeralda, de oro, y adornada de plumería, pura casa
de esmeralda preçiada, trasparante como un cristal, de diuersas colores de preciada plumería, y
en ella e de tener aues de diuersas colores de preçiada plumería, a la bista muy suabes y estima-
das, y asimismo tener y poseer géneros de preçiadas maçorcas y cacao de muchas colores; asi-
mismo tener todas suertes de colores de algodón y hilados. Todo lo tengo de beer y tener, pues
me es mandado y mi ofiçio, y a eso bine.

(Tezozomoc 2001: 56-57)

Die diphrastische Struktur irritiert bisweilen den chronologischen Ablauf der erzählten Ereignisse, etwa wenn die Abfolge der Orte, die die Mexica durchwandern, von der Linearität in die Doppelformel übertragen wird: „,Y, salidos de Ocopipilla y Acahualçinco, partieron de allí y binieron a la parte que llaman Coatepec [...]“" (Tezozomoc 2001: 57). Tezozomoc erzählt nicht, wie bei einer linearen Geschichtskonzeption zu erwarten wäre, erst alles, was in Ocopipilla passiert und dann alles, was im nächsten Ort der Wanderung, Acahualçinco, geschieht, um danach zur nächsten Station Coatepec überzugehen, sondern er fasst die beiden Orte Ocopipilla und Acahualçinco in einer Diphrase zusammen, und durchbricht so die Chronologie des Geschehens. 
Redundanzen durch Parallelismen sind ein weiteres Mittel, das Tezozomoc einsetzt, um den Eindruck oraler Überlieferung herzustellen. Der Erzählstill der Crónica Mexicana erweist sich dadurch als eher additiv statt chronologisch-kausal.

Y de allí salieron y llegaron al pueblo que es agora de Atlitlalaquian, que es Atitalaquia, pueblo de otomíes. Y de allí binieron a Tequixquiac y allí labraron camellones y llamáronle chinamitl, que oy permanesçe este bocablo en Nueua España. Y de allí binieron y llegaron en Atengo, y allí pusieron el tzompan, un término de cantidad, y así se le quedó el lugar, que agora es pueblo de Çumpango. Y de allí binieron y llegaron a Cuachilco, y de allí a Xaltocan, caminando ya poco a poco y de poca distançia. Y allí en Xaltocan hizieron camellones dentro del lago (chinamitl), sembraron maíz y huauhtli, frisol, calabaça, chilchotl, xitomate. Y de allí en po<co $>$ s años caminaron y llegaron en Eycoac (En la parte de las tres culebras), asimismo hizieron sus sementeras y sembraron. Y de a po<co $>$ s años llegaron a Ecatepec, y de allí se abían diuido en Acalhuacan. Y de allí se binieron a Tulpetlac. Y de allí se binieron a Huixachtitlan. Y de allí binieron a Tecpayuca.

(Tezozomoc 2001: 61)

Tezozomoc arbeitet darüber hinaus mit Epitheta, die von Ong (1987: 43) als Teil des „formelhafte[n] Gepäck[s]“ bezeichnet werden, das die Funktion habe, das Denken im Rahmen der mündlichen Überlieferung zu stabilisieren. So ist nicht einfach nur von Huitzilopochtli die Rede, sondern von „Huitzilopochtlis, ydolo dios de ellos“ (Tezozomoc 2001: 53), „el demonio Huitzilopochtli““ (ibd.: 54), ,el dios Huitzilopochtli“ (ibd.: 56) oder „gran diablo Huitzilopochtli““(ibd.: 58).

Weiterhin nennt der Geschichtsschreiber Respektsnamen für Herrscher oder sozial höhergestellte Personen, deren wörtliche Übersetzung er angibt, ohne jedoch den genauen metaphorischen Sinn oder die konkrete Verwendungsweise in bestimmten Sprechsituationen zu erläutern: „y por consuelo dize el más biexo saçerdote: ,Rrey y señor, niño, cozcatle (preçiado collar de fina piedra), preçiosa pluma rrica $n<$ uest>ra (toquetzale), nieto $n<$ uest $>$ ro tan querido [...]““(Tezozomoc 2001: 234; cf. Graham Classen 1998: 124).

Den Beinamen Tlacaeleltzins erläutert Tezozomoc so: „E luego se puso y adereçó Atenpanecatl, prençipal, a la mensajería de parte de los mexicanos, que por tener el rrenombre de Tlacaheletzin se atrebió, como dezir Gran barón de mucha cólera, prudencia y rrazón“ (Tezozomoc 2001: 72). Für einen europäisch geprägten Leser könnte die Nennung von cólera im Sinne einer positiv konnotierten Eigenschaft ungewöhnlich erscheinen. Es könnte sich aber um eine Übersetzung des Namensbestandteils elel handeln, der sich von elli ,Leber ' herleitet, was als der Sitz der Emotionen galt; zudem könnte cólera mit ,Mut‘ zusammenhängen (cf. Graham Classen 1998: 123).

Der bereits erwähnte additive Charakter der Chronik wird durch die parataktische Syntax verstärkt. Das Verfahren des Polysyndetons, etwa durch die Häufung der Konjunktion $y$, dominiert, wie aus den folgenden Beispielen hervorgeht:

[P]orque el día que llegaron en esta laguna mexicana en medio della estaua y tenía un sitio de tierra y en él una peña y ençima de ella un gran tunal; y en la ora que llegaron con sus balsas de caño y carrizo hallaron en el sitio la $\mathrm{d}<\mathrm{ic}>$ ha piedra y tunal y al pie dél un hormiguero, y estima ençima del tunal una águila comiendo y despedaçando una culebra; y así tomaron el apellido y armas y diuisa, el tunal y águila, que es tenuchca o tenuchtitlan, que oy se nombra así.

(Tezozomoc 2001: 54) 
<que> heran de siete barrios, cada uno de su barrio traía el nombre de su dios, como era Quetzalcoatl, Xocomo y Matla, Xochiquetzal y Chichitic, Çentutl y Piltzinteuctli, Meteutl y Tezcatlypuca, Mictlanteuctli y Tlamacazqui y otros dioses, que aunque cada barrio de los siete traía señaldo su dios, traían asimismo otros dioses con ellos, y los que más hablan con los yndios eran Huitzilopuchtli y Tlacolteutl y Mictlanteuctli. El uno de los barrios se llamaua Yopica y Tlacochçalca y el tercero barrio Huitznahuac y Çihuatecpaneca y Chalmeca y Tlacatecpaneca, y el seteno barrio se llaman Yzquiteca.

(Tezozomoc 2001: 55)

Weitere Annäherungen an die mündliche Sprache werden durch Inversionen hergestellt: „La benida que hizieron y tiempos y años que estubieron en llegar a este Nueuo $\mathrm{Mu}<\mathrm{n}>\mathrm{do}$, adelante se dirá“ (Tezozomoc 2001: 53), ,y luego alçauan el sarzo por mandato de su dios Huitzilupochtli, les hablaua y ellos rrespondían y luego a su mandato, les dezía“ (ibd.: 54); „que todas animales ponçoñosas llamaua con ellas hazer muchos males y daños causar muchas muer muchas muertes, çientopiés, arañas ponçoñosas; y usar del arte de bruxa, que se tra $<$ n $>$ sformaua del aue o animal que ella quería“ (ibd.: 56). Der teilnehmende Charakter oraler Darstellungen, wie Ong es formuliert (cf. 1987: 49-50), wird zum Beispiel bei kurzen Dialogen deutlich, die keine Ansprachen darstellen, sondern knappe Zurufe, etwa in der Situation einer Schlacht oder eines Kampfes:

Començaron a caminar por la bía adelante con el exérçito mexicano, aunque muy pocos, y se binieron a topar los dos campos la parte <que> llaman Momoztitlan Tlachtonco. Allí començó a bozear Tlacaeleltzin diziendo: ,¡A ellos, a ellos!' Yban tan furiosos los mexicanos los lleuaron hasta en Tlenamacoyan, <que> yban a más huir los de Cuyuacan, y yban con mucha grita y bozería, apellidando: , $i E a$, mexicanos, agora es!‘

(Tezozomoc 2001: 95)

Es lässt sich feststellen, dass Tezozomoc die mündlichen Überlieferungstraditionen der Mexica durch ganz unterschiedliche diskursive Strategien in seine Chronik integriert. Nicht nur führt er Dialoge und Reden historischer Figuren an, sondern er bemüht sich auch, die Stilmittel und Konventionen der mündlichen Rede der Nahuas zu reproduzieren. Dies scheint bisweilen wichtiger zu sein als die logische Repräsentation der Ereignisse. Tezozomoc geht es also nicht nur um die Überlieferung der historischen Ereignisse. Eine bedeutende Funktion seines Werks liegt vielmehr darin, die Art und Weise, wie die Mexica über die Vergangenheit sprachen, in das Medium der alphabetschriftlichen Chronik zu übersetzen und damit zumindest einen ungefähren Eindruck dessen zu vermitteln, was sich im 16. Jh. mangels technischer Möglichkeiten letztlich nicht aufzeichnen und speichern ließ.

\subsection{Thematisierung und Translation von piktographischen Codices}

Auf piktographisches Material als Quellen für seine Chronik verweist Tezozomoc nicht direkt, sondern im Rahmen wörtlicher Figurenrede. Diese Verweise häufen sich vor allem gegen Ende des Textes, als die Spanier sich auf das amerikanische Festland begeben und es zu den ersten Kontakten mit den Bewohnern Mesoamerikas kommt. Im Kapitel 4.3 wird auf eine solche Passage im Hinblick auf die Hybridisierung des Zeitverständnisses näher eingegangen werden. Im Folgenden soll es zunächst um die Frage gehen, welchen Einfluss die Piktographien strukturell auf die Crónica Mexicana haben.

Durch die Gliederung seines Textes in Kapitel mit je kurzen vorangestellten Inhaltsbeschreibungen scheint sich Tezozomoc an europäische Konventionen zu halten (cf. Dyckerhoff

ISSN 1615-3014 
1970: 14). Bei näherer Betrachtung der Auslöser für Kapitelübergänge jedoch lässt sich diese Beobachtung nicht aufrechterhalten.

So folgt die Kapiteleinteilung zu Beginn des Textes kalendarischen Einschnitten, die für die Mexica von besonderer Bedeutung waren. Der Übergang vom zweiten zum dritten Kapitel wird am Ende einer aztekischen Kalenderrunde (xiuhmolpilli), d. h. eines 52-Jahre-Zyklus (zum Kalendersystem der Nahuas cf. etwa Prem 1990), angesetzt: „Y allí fue fin de años pasados que llaman ,yn xiuhmolpililli yn mexica', como año bisiesto“ (Tezozomoc 2001: 60). Strukturgebend sind hier die aztekische Kalendertradition und das damit verbundene zyklische Zeitverständnis, also gerade nicht die europäische Tradition.

Statt historischer Ereignisse, abgeschlossener Handlungen oder bestimmter Wendepunkte, die historische Texte europäischer Prägung strukturieren (cf. Graham Classen 1998: 41), scheint die Textsegmentierung der Crónica Mexicana bisweilen der Bilderabfolge eines piktographischen Codex zu folgen, wie Graham Classen (1998: 61) hervorhebt: „No se sabe a qué criterio se deben las divisiones de los capítulos en la Crónica Mexicana, pero en apariencia están más ligadas a una lectura directa de las imágenes pintadas en los códices“. So enden Kapitel oft mit visuell repräsentierbaren Ereignissen wie der Ankunft bzw. Abreise von Gesandten (cf. ibd.).

Als Tezozomoc vom Empfang des Herrschers Tizoc in Tenochitlan erzählt (Kap. 59), markiert er am Ende einer zeremoniellen Mahlzeit, das im folgenden Kapitel (Kap. 60) noch einmal aufgegriffen wird, die Kapitelzäsur: „Y luego, acabada la comida [...]“ bzw. „Acabados de comer [...]“" (Tezozomoc 2001: 259). Ausgehend von einem linearen Geschichtsverständnis, das eine chronologische Abfolge von Ereignissen präsentiert, erscheint diese Struktur des Textes redundant, da der Kapitelübergang an einem eher nebensächlichen Punkt erfolgt und dieser auch noch wiederholt wird. Betrachtet man die Passage jedoch aus der Perspektive eines Betrachters von Piktographien, so wird der visuelle Charakter, der die Textstrukturierung leitet, deutlich: Während die Abschlussszene das Essen beinhaltet, wird Tizoc zu Beginn des nächsten Kapitels neu eingekleidet, d. h. ein neuer Abschnitt im ritualhaften Empfang des Herrschers setzt ein. Wichtiger als politische Ereignisse ist das diplomatische Protokoll mit all seinen Details. Hier wird noch einmal der eingangs erwähnte Doppelcharakter der Crónica Mexicana deutlich, die historische wie auch ethnographisch relevante Daten liefert.

Der Tod Tizocs, der im 61. Kapitel stattfindet, wird beinahe beiläufig erzählt: „Y permitió la magestad ymmensa diuina que antes que este moço rrey tantas crueldades usase, murió y allá fue con Huitzilopochtli“" (Tezozomoc 2001: 262). Die Kapitelzäsur erfolgt jedoch nicht bei diesem politischen Ereignis, sondern bei der Ankunft der beiden anderen Herrscher der aztekischen Dreibundpartner, Tetzcoco und Tlacopan, und den sich daran anschließenden Trauerritualen. Wieder hat die Beschreibung der diplomatischen Sitten strukturell Vorrang vor den eigentlichen Ereignissen. Nicht die Herrschaftszeiten oder die Abfolge der Regenten, wie es in der europäisch geprägten Geschichtsschreibung zu erwarten wäre, strukturieren die Erzählung, sondern visuell repräsentierbare Geschehnisse.

Ein weiteres Beispiel dafür sind die politischen Gesandtschaften, die zwischen verschiedenen Herrschern vermitteln und die von Tezozomoc nicht nur namentlich erwähnt werden, sondern deren Ankunft, Dialoge und Abreise er detailliert beschreibt (cf. Graham Classen 1998: 38). So nimmt bspw. im achten Kapitel der Crónica Mexicana die Auswahl des Gesandten an die 
Tepaneken viel mehr Raum ein als das Ereignis, das die Gesandtschaft überhaupt erst notwendig machte: die Ermordung des mexicanischen Herrschers (Chimalpopoca). Dagegen bestimmt die Auswahl des Gesandten und dessen Gesprächsvermittlung zwischen Mexica und Tepaneken über die Konsequenzen dieser Tat mehrere Kapitel. Die politische Kommunikation zwischen den Parteien erscheint so als das bedeutendere Element im Vergleich zu den politischen Ereignissen.

In der Crónica Mexicana dominiert die Gliederung der Geschichte in Szenen vor einem durchgehenden chronologisch-linearen Aufbau (cf. Graham Classen 1998: 62). Ereignisse werden in Form von Dialogen oder durch die detailreiche Beschreibung von Handlungen präsentiert, ohne dabei unbedingt narrative Verknüpfungen (z. B. durch Kausalbeziehungen) oder Erklärungen zu liefern. Tezozomoc äußert sich nicht zu den Gedanken oder Gefühlen der Figuren, die er sprechen lässt (cf. Graham Classen 1998: 64). Die Dialoge zwischen historischen Akteuren führen auch nicht unbedingt zu einer multiperspektivischen Darstellung der Geschichte, denn Tezozomoc verlagert oft nur seine eigenen Ansichten in die Figurenrede.

Durch die Konzentration auf die Details der politischen Rituale, die umfangreichen Dialoge, das Fehlen von Abstraktionen und Verdichtungen sowie von Emotionen und Innensichten der involvierten historischen Figuren wird weder innerhalb einer Szene noch übergreifend im gesamten Text eine einheitliche Erzählerperspektive etabliert (cf. Graham Classen 1998: 7181). Tezozomoc konzentriert sich damit weniger auf die Ordnung des Geschehens als vielmehr auf die Momente als solche, mit ihren visuellen, räumlichen und auditiven Elementen, d. h. er präsentiert keinen einheitlichen Ablauf, sondern reiht Handlungssequenzen aneinander, vergleichbar mit den Bildsequenzen eines piktographischen Codex: „En Tezozómoc hay varios enfoques o núcleos de acción cuyo resultado es una estructura narrativa en escenas. [...] Es una narración más ligada a los detalles de la escena pictográfica“ (ibd.: 88-89). Auch Cortés (2006: 29) kommt daher zu dem Schluss, dass die Piktographien als eine Art „guía recordatoria“ für das Verfassen der Crónica Mexicana angesehen werden können.

Diese Erzählstruktur führt zwar dazu, dass die Kausalität der historischen Ereignisse sowie die Motivation der Akteure in den Hintergrund rücken. Tezozomoc gelingt es jedoch, durch die Kombination der szenisch-piktographischen Textstruktur mit ausführlichen fiktionalisierten mündlichen Passagen die Konventionen aztekischer Geschichtsüberlieferungen in das Medium der alphabetschriftlichen Chronik zu übersetzen. Von einer Hybridisierung kann deshalb gesprochen werden, weil das aztekische Substrat der Crónica Mexicana in der Erzählstruktur selbst sichtbar bleibt, auch wenn Tezozomoc sein Werk auf Spanisch verfasst und als schriftlichen Text konzipiert hat, wie aus einer der wenigen metanarrativen Passagen hervorgeht: „,[O]raçión muy eroica, que por no cansar al lector tan larga prolixidad no la escribo“ (Tezozomoc 2001: 256).

\subsection{Hybridisierung des Zeitverständnisses}

Nach der Auseinandersetzung mit transmedialen Übersetzungprozessen zwischen Mündlichkeit, Piktographie und Alphabetschriftlichkeit stellt sich nun die Frage, inwiefern auch hinsichtlich des Geschichtskonzepts bzw. Zeitverständnisses in der Crónica Mexicana von einer Hybridisierung ausgegangen werden kann. Ich möchte mich dieser Frage annähern, indem ich noch einmal auf das Thema der Piktographien zurückkomme und ausgehend von

ISSN 1615-3014 
einem Beispiel analysiere, wie der chronologisch-lineare Ablauf der Geschichte der Mexica nicht nur durch die Einteilung in Szenen irritiert wird, sondern auch durch die der Chronik zugrundeliegenden Metaerzählung.

Gegen Ende der Crónica Mexicana beschreibt Tezozomoc die Versuche Moctezumas und seiner Untergebenen, zu verstehen, wer die Spanier sind und wie ihre Ankunft zu deuten ist. Noch ist es nicht zu einer direkten Begegnung gekommen. Ein großer Weiser (gran sabio) namens Quilaztli wird beauftragt, Moctezuma diese Fragen zu beantworten. Er findet die Antwort in einem alten Codex, die von der Ankunft von auf Schlangen reitenden Gottesträgern (teomamaque) berichtet, die über das Meer aus dem Osten kämen und die Herrschaft über Mesoamerika übernehmen würden:

Bernán luego otros de un pie y an de benir otras gentes que no tienen cabeças sino <en> los pechos cabeça, cara y boca. [...] Y an de benir por Tzonapan, por çima de la Gran Mar, muy blancos de rrostro y todo el cuerpo y de muy largas baruas y los bestidos de muchas y diferentes manera y de muchas colores.' [...] Començó de enmudeçer Monteçuma y llorar amargamente $[\ldots]$

(Tezozomoc 2001: 477-478)

Tezozomoc verknüpft in dieser Passage das zyklische Zeitverständnis der Mexica mit europäischen Referenzen und Deutungen vom Ende des Aztekenreiches.

Der bekannteste Mythos Mesoamerikas, der das zyklische Zeitverständnis thematisiert, ist sicher die sog. Leyenda de los soles, der zufolge die Weltgeschichte in fünf Epochen strukturiert ist, die jeweils mit einer verheerenden Naturkatastrophe endeten und die Menschheit vernichteten (eine Übersicht der verschiedenen Varianten dieses Mythos liefert Moreno de los Arcos 1984).

Da die Crónica Mexicana die mythische Vorzeit Mesoamerikas nicht bespricht, sondern erst mit dem Auszug der Mexica aus Aztlan bzw. Chicomoztoc einsetzt, wird der SonnenzeitalterMythos nicht thematisiert. Zyklisches Zeitverständnis wird stattdessen in die politische Geschichte integriert. Tezozomoc zufolge muss nämlich jedes Reich aufgrund der Arroganz des Herrschers unweigerlich nach einer bestimmten Zeit zugrunde gehen, wie er am Beispiel von Moctezuma zeigt.

Die Ankunft Cortés' wird in diese zyklische Vorstellung eingebaut, indem Quilaztli aus dem Codex das Ende der Herrschaft Moctezumas als alte Überlieferung herausliest. Der Regent signalisiert durch seine Trauer, dass er diese Deutung akzeptiert. Ein möglicher Einwand wäre, dass es sich hier schlicht um eine rückblickende Legitimierung der spanischen Eroberung handelt. Die Interpretation des Codex durch Quilaztli ist zudem, obwohl sie die aztekische Tradition widerzuspiegeln scheint, von Europäismen durchsetzt. So prognostiziert der Codex außerdem die Ankunft von kopflosen Menschen, sog. Azephalen. Dies erinnert an europäische Legenden, Naturgeschichten und Reiseberichte der Antike (z. B. bei Plinius) und des Mittelalters (etwa Mandeville oder Polo), die derartige monströse Menschen in Asien vermuteten. Dies war noch zur Zeit der europäischen Expansion ein so stark etabliertes Wissen, dass Colón sich in seinem Brief an Santángel 1494 genötigt sieht, darauf hinzuweisen, bisher in Amerika nicht auf dergleichen gestoßen zu sein (cf. Colón 2000: 48). Bei Tezozomoc werden die monströsen Menschen, die von den europäischen Eroberern in Amerika vermutet wurden, in eine mesoamerikanische Prophezeiung überführt und auf die Spanier übertragen.

ISSN 1615-3014 
Auch in der europäischen Tradition ist übrigens mit dem Konzept der „translatio imperii“ ebenfalls ein Geschichtskonzept mit einer zyklischen Komponente bekannt. Auch hier ist das Motiv des „schuldhaften Mißbrauchs der Herrschaft“ (Curtius 1993: 39) geläufig. Die politische Deutung der ursprünglich biblischen Lehre erfolgte im Mittelalter (cf. Curtius 1993: 38). Assmann (1999: 111-112) erläutert, dass die „translatio imperii“ in Europa eng an den imperialen Mythos gebunden ist, wobei die Idee einer universalen Kultur besteht, die durch die Abfolge der Reiche hindurch tradiert wird. Die Figur der „translatio“ bestimmt er wie folgt: Sie

rechnet mit dem Untergang von Kulturen und sichert entsprechende Anschlußstellen: das Licht, das hier zu ende geht, kann dort wieder angezündet werden. Sie rechnet jedoch nicht mit den Intervallen dunkler Zwischenzeiten, in denen das Kontinuum der Kultur unterbrochen ist.

(Assmann 1999: 115)

Gleichwohl sind mögliche Anleihen aus der europäischen Tradition bei Tezozomoc nur ein Aspekt. Auch aus dem Verlauf der aztekischen Geschichte kann ein zyklisches Zeitverständnis abgeleitet werden. Dieses wird von Tezozomoc nicht explizit thematisiert. Es liegt der Crónica Mexicana jedoch implizit als Erklärungsmodell zugrunde, warum die Mexica nach dem Fall Tollans das von Huitzilopochtli auserwählte Volk sind und warum nach ihrem Aufstieg zur führenden Macht Zentralmexikos der Fall Tenochtitlans doch unvermeidlich ist (cf. Cortés 2006: 29).

Es geht aus dem Text dadurch hervor, dass der Niedergang des Aztekenreiches aus dem Modell des Niedergangs des von Quetzalcoatl bzw. Huemac beherrschten Tollan erklärt wird (cf. Cortés 2006: 31). In beiden Fällen gehen der Niederlage eine Reihe von Vorzeichen voraus, die ein außergewöhnliches Ereignis ankündigen sollen. Eines Tages etwa sieht Moctezuma eine weiße Wolke in den Himmel aufsteigen:

Y lo que significó fue, estando arando un yndio en el çerrillo de Quetzaltepetl, bino una águila caudal y sin sentirlo ni berlo el yndio, le asió de los cabellos y lo lleuó ençima de un çerro alto y de súpito lo metió <en> una sala, el mejor que jamás biera, y la propia águila no bio, sino un prençipal gran señor. Díxole: 'Ben acá, no tengas temor. Toma esta rrosa y este perfumador, huélgate. Pero mira quál está aquí tendido Monteçuma borracho perdido, que no sabe de sî́.

(Tezozomoc 2001: 453)

Der prençipal gran señor befiehlt dem macehual (also einem Nichtadligen), Moctezuma zu verletzen, damit er erkenne, dass dieser nicht bei Sinnen ist. Dann trägt er ihm auf, dem Herrscher persönlich sein Erlebnis zu erzählen, was er auch tut. Hier nutzt Alvarado Tezozomoc das für den mündlichen Diskurs typische Verfahren, bereits Erzähltes noch einmal zu wiederholen, statt, wie im alphabetschriftlichen Diskurs üblicher, sich des Stilmittels der Ellipse zu bedienen. Moctezuma verurteilt den macehual zum Tode, da er ihn, als er die Schmerzen der Verletzung zu spüren beginnt, für einen Zauberer hält (cf. Tezozomoc 2001: 455). Moctezuma schickt Gesandte zu Huemac, dem Herrscher von Cincalco (einer Höhle in der Unterwelt, cf. ibd.: 461: „cueba ynfernal“) und ehemaligen Regenten von Tollan. Huemac kritisiert Moctezumas Regierungs- und Lebensweise: „Pues quiero <que > sepa que es pobre y él propio se lo quiso y lo buscó <en> la manera de su biuir. Y es que ya está d<ich >o y nonbrado su propio nombre, que ello fue demasiada soberuia y crueldad suya ynhumana con sus próximos" (ibd.: 459). Moctezuma selber ist es, der sein Ende zu erahnen scheint (cf. Cortés 2006: $33)$ : 
Hijos, ya e hallado a dónde abemos de yr y todos bosotros comigo, que es en Çincalco. Y emos de estar <en> conpañía del que andaua ya a muchos años a Tula, que nos truxo aquí, que se llama Huemac. Y si allá <en>tramos, jamás moriremos, sino biuir para siempre adonde ay quantos géneros de comida ay en el mundo y beuidas y todo género de rrosas y todo género de árboles frutales, porque todos los moradores que allá, están los más contentos del mundo [...].

(Tezozomoc 2001: 455)

Allerdings wird er von Huemac über den Charakter der Unterwelt korrigiert, wodurch er wiederum als unbesonnener Herrscher kritisiert wird: „Agora es todo tormento, que no es este lugar como allá el rrefrán dizen ques, un deleitoso paraíso de contento, sino un continnuo tormento. Dezilde esto a Monteçuma, que si biese este lugar de puro temor huyera hasta meterse en una dura piedra“ (Tezozomoc 2001: 458). Bereits vor Moctezumas Versuchen, von Huemac Rat und Hilfe einzuholen, hatte Nezahualpilli angedeutet, dass der Herrscher Tenochtitlans nicht mehr mit dem Wohlwollen der Götter rechnen könne (cf. Cortés 2006: 32) und sein Ende angesichts der Vorzeichen feststehe:

Si es ya así la boluntad de n<uest>ros dioses que esto se acabe, ¿qué puedo yo dezir? Lo que os rruego y <en>cargo como baleroso hombre de buen pecho y de gran coraçón que os esforçéis y cobréis ánimo baleroso, ynbençible, de rresçibir estos golpes de fortuna, pues es ya permisión que esto se acabe.

(Tezozomoc 2001: 441)

Die Parallele zwischen Moctezuma und Huemac wird in der Crónica Mexicana nur angedeutet, weil Alvarado Tezozomoc die Herrschaft Huemacs nicht eigens behandelt. Zieht man jedoch die Leyenda de los soles heran, so stellt sich heraus, dass Huemacs Regentschaft ähnlich defizitär war wie die Moctezumas: Als Huemac den Sieg bei einem Ballspiel davontrug, verlangte er als Prämie Quetzalfedern und Jade statt der Maiskolben, die ihm angeboten wurden. Diese Bevorzugung von Reichtümern gegenüber Lebensmitteln führte zu jahrelangen Missernten bei den Tolteken und schließlich dazu, dass Huemac die Herrschaft verlor und nach Cincalco fliehen musste (cf. Cortés 2006: 33). Tollan wurde daraufhin an die Mexica übergeben, wie sich Moctezuma wohl bewusst ist: „que nos truxo aquí, que se llama Huemac“ (Tezozomoc 2001: 455).

Die Übertragung der Herrschaft über Tollan an die Mexica erwähnt Alvarado Tezozomoc im dritten Kapitel der Crónica Mexicana (Cortés 2006: 32): „Y así, llegaron al pueblo que es agora de Tula que, asegún otros dizen, allí abían estado y permanesçieron y señorearon con los de Tula beinte y dos“ (Tezozomoc 2001: 61). In den Anales de Cuauhtitlan wird von Quetzalcoatl berichtet, dass er, ähnlich wie Huemac gemäß der Leyenda de los soles, aufgrund seines Fehlverhaltens Tollan verlassen muss: Er lässt sich von seinem Gegenspieler Tezcatlipoca zum Alkoholgenuss überreden und begeht daraufhin Inzest mit seiner Schwester (cf. Cortés 2006: 34). Alvarado Tezozomoc greift also mit seiner Darstellung Moctezumas auf ein rekurrentes Motiv in der aztekischen Überlieferung zurück: Das individuelle Fehlverhalten der Herrscher führt unweigerlich zum Untergang des jeweiligen Reiches - „el paradigma cíclico de destrucción y restablecimiento del orden da coherencia a la historia mexica pata entender la pérdida del imperio en la Crónica Mexicana“ (Cortés 2006: 35).

Man könnte einwenden, dass diese Darstellung auch als Legitimation der spanischen Kolonialherrschaft interpretierbar sei, da der Untergang von Moctezumas Reich als unvermeidlich erscheint, worauf auch Cortés (2006: 38) hinweist. Andererseits kommen die Spanier bei Al- 
varado Tezozomoc als Akteure kaum vor, da die Crónica Mexicana endet, bevor es zur ersten Begegnung zwischen Cortés und Moctezuma teuczoma kommt. Die Geschichte ist auf die Mexica konzentriert; diese stehen als Akteure im Zentrum, und aus ihrer mythisch eingebetteten Geschichte heraus, ohne das Eingreifen eines christlichen Gottes o. ä., entwickeln sich die Ereignisse. Die Handlungsmacht oder gar Überlegenheit der Spanier ist nicht Teil dieses Narrativs. So wird keine universalisierende Deutung der Ereignisse über die mexikanischen Vorstellungen hinaus etabliert - Alvarado Tezozomoc konzentriert sich auf die Darstellung der Handlung der einzelnen Personen (cf. Graham Classen 1998: 50), etwa die detaillierte Beschreibung der drei Gesandtschaften, die Moctezuma zu Huemac schickt. Damit ist die Unabänderlichkeit des Niedergangs des Aztekenreiches zugleich unhinterfragt und keine besondere Leistung der Spanier, da der Übergang von einer Macht zur anderen Alvarado Tezozomoc zufolge schon a priori in der mesoamerikanischen Geschichte angelegt ist.

Zieht man dazu noch das europäische Konzept der „translatio imperii“ aufgrund der moralischen Korruption der Herrscher heran, so verstärkt sich der Eindruck, dass Tezozomoc zumindest andeutungsweise auch die Dauer der Herrschaft der Spanier hinterfragt. Das zyklische Geschichtsverständnis kann also eventuell auch als versteckte Relativierung der (in der Crónica Mexicana nicht erzählten) spanischen Hegemonie gelesen werden.

\section{$5 \quad$ Fazit}

In der Crónica Mexicana sind unterschiedliche Überlieferungsformen miteinander verschränkt. Der Text richtet sich also eigentlich an einen Leser, der mit beiden kulturellen Welten vertraut ist (cf. Lienhard 1990: 150). Zwar werden Informationen für die mit der aztekischen Geschichte weniger vertrauten Leser geliefert und es werden Ausdrücke aus dem Nahuatl übersetzt, die Darstellungsweise und Diegese kommen aber teilweise auch aus der aztekischen Tradition (cf. ibd.: 184-185). Beide Bereiche finden jedoch nicht unbedingt zu einem harmonischen Ganzen; so stellt Lienhard fest, dass die eine Tradition die Arbeit der anderen behindert (cf. ibd.: 185).

Daher wäre es verkürzt zu sagen, die Crónica Mexicana stünde für eine Europäisierung, nur weil sie auf Spanisch verfasst ist (cf. Lienhard 1990: 186). Nur ein bilingualer und bikultureller Leser könne, so Lienhard, den Text wirklich erfassen, der sich deshalb weder als spanischer noch als indigener, noch auch als mestizischer Text erfassen lasse - für letzteres sei er nicht kohärent genug, es sei denn, man versteht „,mestizisch“ als die Sphäre des Hybriden: „Si lo ,mestizo“, en cambio, es la esfera de lo ,híbrido“, del conflicto entre los sistemas de signos autóctonos y los de origen europeo, este texto sería un excelente ejemplo para ilustrarlo“ (ibd.: 186).

Durch die Abfassung der Chronik in spanischer Sprache kann der Leser sie in die „universale“, christliche Kultur integrieren; zugleich bleibt die ,aztekische“ (partikulare) Stimme erhalten - damit leistet Tezozomoc, ob bewusst oder unbewusst, „una transformación de las reglas de homogeneización cultural que rigen la literatura ,universal' de su momento: ésta debería asumir la pluralidad cultural“ (Lienhard 1990: 187). 


\section{Literatur}

Alvarado Tezozomoc, Hernando (2001): Crónica Mexicana. Herausg. von Germán Vázquez Chamorro und Gonzalo Díaz Migoyo. Madrid: Dastin.

Alvarado Tezozomoc, Hernando (2004): Crónica Mexicayotl. Herausg. Von Berthold Riese Sankt Augustin: Academia.

Assmann, Aleida (1999): Zeit und Tradition. Kulturelle Strategien der Dauer. Köln etc.: Böhlau.

Barlow, Robert Hayward (1945): «La Crónica X: versiones coloniales de la historia de los mexica tenochca». Revista Mexicana de Estudios Antropológicos 7/1-3: 65-87.

Batalla Rosado, Juan José (2008): «Los códices mesoamericanos: métodos de estudio». Itinerarios 8: 43-65.

Carrasco, Pedro (1996): Estructura político territorial del Imperio tenochca: la triple alianza de Tenochtitlan, Tetzcoco y Tlacopan. Ciudad de México: FCE.

Colón, Cristóbal [Kolumbus] (2000): Der erste Brief aus der Neuen Welt. (Spanisch/Lateinisch/Deutsch). Herausg. und übers. von Robert Wallisch. Stuttgart: Reclam.

Curtius, Ernst Robert (1993): Europäische Literatur und lateinisches Mittelalter. Tübingen/Basel: Francke.

Díaz Migoyo, Gonzalo (1993): «La crónica indígena mexicana: otro aspecto de la 〈visión de los vencidos»». In: García Martín, Manuel (ed.): Estado actual de los estudios sobre el Siglo de Oro. Actas del II Congreso Internacional de Hispanistas del Siglo de Oro. Salamanca, Universidad de Salamanca: 285-288.

Díaz Migoyo, Gonzalo (2001): «Introducción». In: Alvarado Tezozómoc, Hernando: Crónica Mexicana. Barcelona, Dastin: 5-27.

Dyckerhoff, Ursula (1970): Die „Cronica Mexicana“ des Hernando Alvarado Tezozomoc: quellenkritische Untersuchungen. Hamburg: AK Anthropologische Wissenschaften an der Universität Hamburg.

Graham Classen, Ann Marie (1998): Dos interpretaciones de la historia de los mexicas: un análisis comparativo de la Crónica Mexicana de Hernando Alvarado Tezozómoc y la Historia de las Indias de Nueva España e Islas de Tierra Firme de fray Diego Durán. Ciudad de México: UNAM.

Gruzinski, Serge (1993): The Conquest of Mexico. Cambridge: Polity Press.

Hill Boone, Elisabeth (2000): Stories in Red and Black. Austin: University of Texas Press.

Lienhard, Martin (1990): La voz y su huella. Escritura y conflicto étnico-social en América Latina (1492-1988). La Habana: Casa de las Américas.

León-Portilla, Miguel (1983): «〈Cuícatl〉 y 〈Tlahtolli〉. Las formas de expresión en náhuatl». Estudios de cultura náhuatl 16: 13-108.

Lockhart, James (1991): Nahuas and Spaniards: Postconquest Central Mexican History and Philology. Stanford: Stanford University Press.

Lockhart, James (1992): The Nahuas after the Conquest: A Social and Cultural History of the Indians of Central Mexico, Sixteenth through Eighteenth Centuries. Stanford: Stanford University Press.

Máynez, Pilar (2002): El calepino de Sahagún: un acercamiento. Ciudad de México: FCE.

Moreno de los Arcos, Roberto (1984): «Los cinco soles cosmogónicos». In: Stavenhagen, Rodolfo (ed.): Imagen y obra escogida. Ciudad de México, UNAM: 15-46. 
Ong, Walter J. (1987): Oralität und Literalität: die Technologisierung des Wortes. Übers. von Wolfgang Schömel. Opladen: Westdeutscher Verlag.

Pardo López, José Manuel/Peralbo Pintado, José Antonio/Torres Jara, Segio Daniel (2002): «Los códices mesoamericanos prehispánicos». Signo. Revista de Historia de la Cultura Escrita 10: 63-91.

Pastrana Flores, Miguel (2004): Historias de la conquista. Aspectos de la historiografía de tradición náhuatl. Ciudad de México: UNAM.

Richter, Annegret (2015): Geschichte und Translation im kolonialen Mexiko. Eine Untersuchung ausgewählter historischer Schriften von Fernando de Alva Ixtlilxochitl, Diego Muñoz Camargo und Hernando Alvarado Tezozomoc. Hildesheim: Olms.

Riese, Berthold (2004): „Einleitung“. In: Alvarado Tezozomoc, Hernando (ed.): Crónica Mexicayotl. Sankt Augustin, Academia: 13-31.

Romero Galván, José Rubén (2003): Los privilegios perdidos. Hernando Alvarado Tezozómoc, su tiempo, su nobleza y su Crónica Mexicana. Ciudad de México: UNAM.

Romero Galván, José Rubén (2007): «Memoria, oralidad e historia en dos cronistas nahuas». Estudios de Cultura Náhuatl 38: 165-182.

Schroeder, Susan (2011): "The truth about the Crónica Mexicayotl". Colonial Latin American Review 20/2: 233-247. 